\title{
Idiopathic multicentric Castleman disease: pathogenesis, clinical presentation and recommendations for treatment based on the Castleman Disease Collaborative Network (CDCN)
}

\author{
Beata Ostrowska , Joanna Romejko-Jarosińska, Katarzyna Domańska-Czyż, Jan Walewski \\ Department of Lymphoma, Maria Sklodowska-Curie National Research Institute of Oncology, \\ National Science Centre, Warsaw, Poland
}

\begin{abstract}
Castleman disease is a very rare, lymphoproliferative disease, driven by dysregulation of the cytokine interleukin 6 (IL-6) and other proinflammatory cytokines with the development of symptoms of systemic inflammation, reactive proliferation of lymphocytes, and damage to numerous organs. HHV-8 infection plays a major role in the pathogenesis of human immunodeficiency virus (HIV+) multicentric Castleman disease (MCD). The aetiology of the idiopathic form of MCD (HIV/ /HHV-8 negative) is unknown.

In 2017, a panel of Castleman Disease Collaborative Network (CDCN) experts developed unified diagnostic criteria necessary for the diagnosis of idiopathic MCD. The disease has a diverse course, with a broad spectrum from mild to severe. The drug of choice according to the CDCN recommendation of 2018 for patients requiring treatment is anti-IL-6 monoclonal antibody, siltuximab. Immunomodulatory and immunosuppressive drugs are an alternative first-line treatment when anti-IL-6/IL-6R drugs are unavailable, and in subsequent lines of treatment after the failure of anti-IL-6 antibodies. Chemotherapy is reserved for the treatment of severe forms of the disease. The prognosis in most patients is moderately good. Unfortunately, the availability of the new therapies remains limited.
\end{abstract}

Key words: multicentric Castleman disease, interleukin 6, siltuximab

Acta Haematologica Polonica 2021; 52, 1: 29-37

\section{Introduction}

Castleman disease $(C D)$ is a very rare, non-neoplastic lymph node hyperplasia of unknown aetiology. Excessive release of interleukin 6 (IL-6) and other pro-inflammatory cytokines results in systemic inflammation, reactive lymphocyte proliferation, and organ damage. CD was first described by Benjamin Castleman in 1954 as angiofollicular lymph node hyperplasia limited to one nodal group, now classified as unicentric Castleman disease (UCD) [1]. Similar lesions involving several nodal groups simultaneously, described by Gaba et al. in 1974, usually accompanied by systemic symptoms, are now diagnosed as multicentric Castleman disease (MCD) [2]. In the 1980s, MCD diagnosis was correlated with immune deficiencies, mainly related to human immunodeficiency virus (HIV) infection. Since then, HIV-associated MCD cases and those unrelated to HIV infection (HIV-) have been systematically distinguished [3]. MCD has been also associated with POEMS syndrome (polyneuropathy, organomegaly, endocrine disorders, monoclonal gammopathy and skin lesions). The discovery of a direct link between HIV-associated MCD and human
*Address for correspondence: Beata Ostrowska, Department of Lymphatic System Cancer, Maria Sklodowska-Curie National Research Institute of Oncology, National Science Centre, Roentgena 5, 02-781 Warsaw, Poland, phone: +48 2254624 48, fax: +48 2254622 23,

e-mail: beata.ostrowska@pib-nio.pl

\footnotetext{
PTH iT copyright @ 2021

The Polish Society of Haematologists and Transfusiologists, Insitute of Haematology and Transfusion Medicine. All rights reserved.
} 
herpesvirus 8 (HHV-8/KSHV, Kaposi sarcoma herpesvirus) infection was a breakthrough in our understanding of the pathogenesis of CD. It has been shown that HHV-8 replicating in plasmablastic cells in germinal centres stimulates the secretion of both viral and human IL- 6 and many other pro-inflammatory proteins, which leads to characteristic histopathological (HP) lesions in the structure of lymph nodes [4]. The discovery of the key role of HHV-8 in the pathogenesis of HIV-associated MCD and the potential role of monoclonal plasma cells in the pathogenesis of POEMS-MCD contributed to the reclassification of CD. The Castleman Disease Collaborative Network (CDCN) has proposed a classification system distinguishing the disease into 1) $C D$ of known or suspected aetiology, i.e. related to HHV-8 infection (HHV8-MCD) often coinciding with HIV infection; 2) cases associated with POEMS (POEMS-MCD); and 3) idiopathic MCD (iMCD) HHV8(-)/HIV(-) of unknown aetiology $[5,6]$. First described in 2010 in Japan, TAFRO syndrome (thrombocytopenia, ascites, reticulin bone marrow fibrosis, renal failure, and organomegaly) has now been recognised as a variant of idiopathic MCD, i.e. iMCD-TAFRO [7]. Patients who do not meet the diagnostic criteria of TAFRO syndrome are classified as having idiopathic multicentric Castleman disease, not otherwise specified (iMCD-NOS) [6].

The incidence of Castleman disease in the general population is unknown. In the United States, approximately 4,750 new cases of $C D$ are diagnosed annually, usually UCD (80-90\%). In multicentric disease, 30-60\% of cases are idiopathic $[6,8]$. The best prognosis is for unicentric disease (UCD). In these cases, surgical resection of the lesion (the treatment of choice) results in long-term diseasefree survival (DFS) in more than $90 \%$ of patients [9]. The prognosis of idiopathic MCD depends on the severity of clinical symptoms. Before the anti-IL6 antibody era, 5-year overall survival (OS) was 55-77\% [10]. The introduction of anti-IL6/IL-6R therapy has significantly improved the quality of life of many patients and prolonged overall survival, especially in the mild form of MCD. Yet the prognosis in the severe disease, including patients with TAFRO syndrome, is still unsatisfactory $[11,12]$. The treatment of HIV-associated MCD patients with a combination of antiretroviral therapy and rituximab has been a great success, increasing 5-year OS from 33\% to 90\% [13].

\section{Pathogenesis of idiopathic multicentric Castleman disease}

$C D$ is believed to result from the dysregulation of the immune system due to the excessive secretion of proinflammatory cytokines. The current pathogenesis model assumes that lymph node hyperplasia and characteristic HP lesions result from the response to an excess of cytokines, mainly IL-6. For several years, the involvement of three main cytokine-stimulating mechanisms has been considered:
1) autoimmune inflammation hypothesis; 2) paraneoplastic syndrome with ectopic cytokine secretion hypothesis; and 3) cytokine stimulation by viruses other than HHV-8 hypothesis $[5,14]$. The relationship between $C D$ and infection with viruses other than HHV-8, including Epstein-Barr virus (HHV-4, human herpesvirus 4) and other viruses from the Herpesviridae family, has not been confirmed [5, 15].

The hypothesis suggesting an autoimmune background highlights the role of germline mutations in immune system genes (innate immunity) and the production of abnormal antibodies. In the lymph nodes, autoantibodies stimulate antigen presenting cells to secrete cytokines [IL-1/tumour necrosis factor alpha (TNF- $\alpha$ )], and presumably could activate yet unknown (MCD-related) cells to overproduce IL-6 and other pro-inflammatory cytokines. In a feedback loop, interleukin 6 may stimulate inflammatory cells to excessive secretion of cytokines (in a kind of vicious circle) [5]. Many autoimmune diseases that develop in consequence of unclear, complex immune system disorders leading to inflammatory response in various tissues and organs, including rheumatoid arthritis (RA), Sjögren's syndrome, systemic lupus erythematosus (SLE), and myasthenia gravis, may coexist with similar histopathological features of lymph nodes as in CD.

According to the currently accepted criteria, several inflammatory and autoimmune diseases have to be excluded in order to diagnose iMCD. Importantly, the presence of antibodies can result from MCD activity only. In approximately $30 \%$ of iMCD cases, anti-nuclear antibodies (ANA), anti-Sjögren-syndrome-related antigen A antibodies (SS-A), anti-platelet or anti-erythrocyte antibodies may be present without the diagnosis of another autoimmune disease $[6,16]$.

An alternative hypothesis suggests that $C D$ is a paraneoplastic syndrome. Somatic mutations lead to the formation of a small pool of monoclonal stromal cells secreting proinflammatory cytokines in the lymph nodes, which become a 'trigger mechanism' that drives cytokine production further. Next Generation Sequencing studies have shown that platelet-derived growth factor beta (PDGFR $\beta$ ) mutations are present in lymph nodes in nearly $20 \%$ of patients with UCD, and the mutations are probably present in the stromal cells (CD45-negative cells) [17]. Analogically to the paraneoplastic POEMS syndrome, in which monoclonal plasmocytes constituting only a small percentage of bone marrow cells are responsible for the excessive secretion of vascular endothelial growth factor (VEGF) and, consequently, to systemic disorders, it has also been shown that patients diagnosed with CD have a higher risk of cancer compared to the general population of a similar age (19\% vs. $6 \%)[5,16]$.

To explain the mechanisms underlying the pathogenesis of MCD, whole-exome sequencing (WES) studies have been performed, which allowed the selection of five unfavourable prognostic genes. Among these, NCOA4 (nuclear receptor coactivator 4 ), also known as ARA70 (androgen 
receptor-associated protein 70$)$, encoding nuclear receptor coactivator 4 protein (NCOA4), is of particular interest.

Abnormal expression and function of NCOA4 has been associated with carcinogenesis. L261F mutation in the NCOA4 gene appears to be highly specific for the idiopathic form of MCD. NCOA4 dysfunction may play an important role in the pathogenesis of CD through its association with MAP kinase (mitogen-activated protein kinase), which controls one of the most important pathways activating IL-6 [18]. Recent studies have suggested that excessive activation of T cells and mTOR protein kinase (mammalian target of rapamycin), associated with the intracellular PI3K/AKT/mTOR signalling pathway, may play a key role in the pathogenesis of iMCD. Early attempts to use mTOR inhibitors in the therapy are promising, and a study with sirolimus is underway in patients resistant to anti-IL6 antibodies [19].

\section{Cytokines and their role in the pathogenesis of Castleman disease}

A key role in the development of $C D$ is attributed to IL-6, a multifunctional cytokine secreted by lymphocytes, macrophages, monocytes, fibroblasts, mesothelial cells, mesangial cells, endothelial cells, as well as numerous neoplastic cells.

To exert biological activity, IL-6 requires binding to the IL-6R receptor (gp-80, glycoprotein-80) on the cell surface (mIL-6R, IL-6 membrane receptor) or in a soluble form (sIL$-6 \mathrm{R}$, soluble IL-6 receptor). Binding with the receptor results in a conformational change of glycoprotein-130 (gp-130), followed by autophosphorylation of gp-130-associated JAK kinase (Janus activated kinase), and phosphorylation of gp-130. The sequence of events leads to the activation of two signal pathways: STAT-3 (signal transducer and activator of transcription 3) and MAPK. In the nucleus, STAT-3 binds to the promoters of the acute phase inflammatory response genes. IL-6 plays an important role in inflammation and regulation of the immune response.

Specifically, it: 1) stimulates B cells and plasma cells to produce immunoglobulins (polyclonal hypergammaglobulinemia); 2) together with IL-1, it activates T cells and antigen-presenting macrophages during acute phase reaction; 3 ) it participates in switching between the innate response (infiltration of neutrophils) and the specific response (infiltration of monocytes and lymphocytes); 4) it stimulates angiogenesis by upregulation of VEGF; and 5) it is involved in the production of acute phase proteins in the liver, including haptoglobin, C-reactive protein (CRP), and hepcidin (decreased production of albumin, iron blocking for haemoglobin) $[5,20]$. IL-6 presumably plays an important role in the development of autoimmune disorders associated with $\mathrm{CD}$ (haemolytic anaemia and immune thrombocytopenia) by stimulating $\mathrm{CD}^{+} \mathrm{B}$ cells to produce autoantibodies. An excess of IL- 6 disrupts the ratio between Th17 cells (excess of pro-inflammatory helper cells) and Treg (regulatory lymphocytes protecting from autoimmunity). The other key cytokines include IL-1, which plays a more significant role in the pro-inflammatory cascade of events than does IL-6. A frequently observed elevated VEGF level is responsible for increased angiogenesis and permeability of blood vessels.

In cases of concomitant POEMS and MCD (POEMS-MCD), excessive VEGF secretion may stimulate the production of IL-6. In other cases (POEMS-like symptoms without the presence of monoclonal plasmocytes), the inverse mechanism is proposed, with stimulation of VEGF secretion by IL- $6[5,14]$. Recently, excessive activation of CD8 ${ }^{+}$ T cells, VEGF-A and the PI3K/AKT/mTOR pathway has been confirmed in patients resistant to IL-6 blockade [19].

\section{Diagnosis, pathomorphology}

HP image of the enlarged lymph nodes is characteristic, but not specific to $C D$. Similar or even identical lesions are described in virtually all enlarged lymph nodes in patients with RA, in approximately $30 \%$ of patients with SLE, and in many other inflammatory and infectious diseases [16]. The ultimate diagnosis requires a comparison between the HP images and clinical symptoms, and the results of laboratory workups with the simultaneous exclusion of inflammation, autoimmune, and neoplastic diseases.

In 2017, the panel of CDCN experts released the first unified diagnostic criteria of iMCD. According to this, two major criteria must be met: typical HP image, and lymph node enlargement $\geq 1 \mathrm{~cm}$ in the short axis in at least two locations. These two major criteria should be accompanied by at least two out of 11 minor criteria (including at least one laboratory criterion). At the same time, it is necessary to exclude all syndromes and diseases overlapping with iMCD (Table I) [6]. There are four HP variants of CD: 1) hyaline vascular (HV), characterised by sclerosis of blood vessels in atrophic germinal centre, concentrically surrounded by small lymphocytes in enlarged mantle zone; 2) plasma cell (PC) with expanded germinal centres and proliferating plasma cells; and 3) mixed variant (MV) with HV and PC features and plasmablastic cell (PB) variant present only in HIV-associated MCD [6].

\section{Clinical presentation}

The idiopathic form of MCD most often affects men aged 40-60 (median 50 years). The plasma cell variant is observed in $40 \%$ of patients, mixed in $40 \%$, and the least common $(<20 \%)$ is the hyaline vascular variant (characteristic for UCD). The most common symptoms of a relatively mild form of iMCD include progressive malaise, fatigue, exercise intolerance, general symptoms (fever, night sweats, weight loss), usually moderate lymphadenopathy (sometimes spontaneous regression of lesions), and 
Table I. Consensus diagnostic criteria for idiopathic multicentric Castleman disease (iMCD) according to Castleman Disease Collaborative Network (CDCN) (2017) [6]

\section{MAJOR CRITERIA (both must be met)}

1. Histopathological confirmation of lesions typical for $C D$, based on lymph node examination

2. Lymphadenopathy $\geq 1 \mathrm{~cm}$ in the short axis, in at least $\geq 2$ areas

MINOR CRITERIA (at least two of these 11 must be met, including at least one laboratory criterion)

\section{Laboratory criteria}

1. $\mathrm{CRP}>10 \mathrm{mg} / \mathrm{L}$ or $\mathrm{ESR}>15 \mathrm{~mm} / \mathrm{h}$

2. Anaemia, $\mathrm{Hb}<12.5 \mathrm{~g} / \mathrm{dL}$ for men and $<11.5 \mathrm{~g} / \mathrm{dL}$ for women

3. Thrombocytopenia, PLT $<150 \mathrm{G} / \mathrm{L}$ or thrombocytosis, PLT $>400 \mathrm{G} / \mathrm{L}$

4. Hypoalbuminemia (albumin $<3.5 \mathrm{~g} / \mathrm{dL}$ )

5. Renal failure (eGFR $<60 \mathrm{~mL} / \mathrm{min} / 1.73 \mathrm{~m}^{2}$ ) or proteinuria (total protein $>150 \mathrm{mg} / 24 \mathrm{~h}$ or $>10 \mathrm{mg} / 100 \mathrm{~mL}$ )

6. Polyclonal hypergammaglobulinemia (lgG $>1,700 \mathrm{mg} / \mathrm{dL}$ )

\section{Clinical criteria}

7. General symptoms: night sweats, fever $>38^{\circ}$, weight loss, fatigue ( $\geq 2$ CTCAE)

8. Enlargement of spleen and/or liver

9. Fluid retention: oedema, ascites, pleural/pericardial effusions

10. Eruptive cherry haemangiomatosis or follicular lesions: violaceous papules

11. Lymphocytic interstitial pneumonia

\section{EXCLUSION CRITERIA (it is necessary to exclude all entities resembling iMCD)}

1. Infectious diseases

HHV-8 (LANA-1 negative in IHC, PCR negative)

EBV-lymphoproliferative disease

CMV, toxoplasmosis, HIV, active tuberculosis

2. Autoimmunological diseases (diagnosis based on applicable criteria for a given disease; presence of autoantibodies is not a criterion that excludes $\mathrm{IMCD}$ )

SLE, RA, Still's disease, juvenile idiopathic arthritis, autoimmune lymphoproliferative syndrome

\section{Neoplastic diseases}

Hodgkin's lymphoma, non-Hodgkin's lymphoma , multiple myeloma, isolated plasmacytoma, follicular dendritic cell sarcoma (FDC sarcoma), POEMS syndrome

CD - Castleman disease; CRP - C-reactive protein; ESR - erythrocyte sedimentation rate; Hb - haemoglobin; PLT - platelets; eGFR - estimated glomerular filtration rate; IgG - immunoglobulin G; CTCAE - Common Terminology Criteria of Adverse Events; HHV-8 - human herpesvirus 8; LANA-1 - latency-associated nuclear antigen; IHC - immunohistochemistry; PCR - polymerase chain reaction; EBV - Ebstein-Bár virus; CMV - cytomegalovirus; HIV - human immunodeficiency virus; SLE - systemic lupus erythematosus; RA - rheumatoid arthritis; FDC - follicular dendritic cell; POEMS - polyneuropathy, organomegaly, endocrinopathy, monoclonal protein, skin lesions

hepatosplenomegaly. Approximately $10-20 \%$ of patients experience more severe symptoms of systemic inflammation, and in addition to 'fatigue syndrome', also vascular leakage syndrome with clinically significant peripheral oedema, fluid accumulation in body cavities (pleura, peritoneum, or pericardium) or symptoms of organ failure (most commonly kidneys and lungs) [11, 16, 21, 22]. In rare cases, the symptoms can be life-threatening.

The diagnostic criteria for severe iMCD have recently been defined by the CDCN. These include organ damage, most commonly kidney disease (eGFR $<30 \mathrm{~mL} / \mathrm{min}$, creatinine $>3.0 \mathrm{mg} / \mathrm{dL}$ ), respiratory failure (interstitial lung inflammation or fibrosis), increased fluid retention (oedema, ascites, pleural or pericardial effusion), severe anaemia (haemoglobin $\leq 8 \mathrm{~g} / \mathrm{dL}$ ) and significant deterioration in performance status [Eastern Cooperative Oncology Group (ECOG) $\geq 2$ ]. A diagnosis requires at least two of these symptoms (Table II). Usually in these cases significant laboratory abnormalities are present, such as high CRP level ( $\geq 100 \mathrm{~g} /$ $/ \mathrm{dL})$, hypoalbuminaemia $(\leq 2.0 \mathrm{~g} / \mathrm{dL})$, and thrombocytopenia $(\leq 100 \mathrm{~g} / \mathrm{L})$ [23].

TAFRO syndrome is a unique form of iMCD. It was first described in 2010 in Japan as a syndrome consisting of thrombocytopenia, ascites, reticulin bone marrow fibrosis, renal dysfunction, and organomegaly (TAFRO = thrombocytopenia, ascites, fibrosis, renal, organomegaly). It is presumed that the spectrum of cytokines involved in the development of TAFRO syndrome may differ slightly from the 
Table II. Diagnostic criteria of severe idiopathic multicentric Castleman disease (iMCD) according to Castleman Disease Collaborative Network (CDCN) (2018) [23]

\section{Severe iMCD symptoms}

1. $E C O G \geq 2$

2. Grade IV renal failure (eGFR $<30 \mathrm{~mL} / \mathrm{min}$, creatinine $>3.0 \mathrm{mg} / \mathrm{dL}$ )

3. Ascites and/or oedema and/or pleural/pericardial effusion

4. Haemoglobin $\leq 8.0 \mathrm{~g} / \mathrm{dL}$

5. Respiratory failure, shortness of breath, symptoms of interstitial lung inflammation or fibrosis

The diagnosis of severe iMCD requires at least two of these five symptoms; ECOG - Eastern Cooperative Oncology Group/ECOG performance status; EGFR - estimated glomerular filtration rate

classical cytokines associated with iMCD-NOS. This explains the thrombocytosis and hypergammaglobulinemia typical for the diagnosis of iMCD-NOS, and the thrombocytopenia and normal or only slightly elevated levels of immunoglobulins observed in TAFRO syndrome. The latter is usually associated with a severe course of disease and a poor prognosis [12].

CD might be associated with paraneoplastic pemphigus (PNP), a very rare symptom often associated with $U C D$, and symptoms resembling POEMS syndrome, including polyneuropathy, present in approximately $2 \%$ of patients [21]. Typical laboratory abnormalities include normocytic anaemia, thrombocytosis or TAFRO-associated thrombocytopenia, high CRP and erythrocyte sedimentation rate (ESR), elevated fibrinogen, polyclonal gammopathy, decreased albumin level, as well as increased IL-6 and VEGF. Bone marrow examination often shows an increased percentage of polyclonal plasmacytes. In the peripheral blood, autoantibodies against erythrocytes and platelets, as well as antinuclear antibodies, are often present [6].

\section{Treatment of idiopathic multiple Castleman disease based on guidelines of CDCN}

The idiopathic form of MCD may be asymptomatic, with mild or moderate systemic symptoms, or immediately life-threatening 'cytokine storm' syndrome leading to multiorgan failure. The therapeutic approach to iMCD varies due to the vast array of symptoms observed in the course of the disease. The first attempt to systematise the diagnostic and therapeutic recommendations was recently made by the CDCN expert panel. In 2016-2018, based on a few published studies and case reports (344 patients) and analysis of the treatment regimens used (479 regimens), the CDCN developed a consensus on three key issues for iMCD. The diagnosis criteria for the severe form of iMCD were defined (Table II), treatment algorithms were proposed (Table III), and treatment response evaluation criteria were developed (Table IV) [23].
Table III. Treatment recommendations for idiopathic multicentric Castleman disease (iMCD) according to Castleman Disease Collaborative Network (CDCN) (2018) [23]

\begin{tabular}{|c|c|}
\hline \multicolumn{2}{|c|}{ iMCD-NOS/IMCD-TAFRO } \\
\hline Mild & Severe \\
\hline \multicolumn{2}{|c|}{ First-line therapy } \\
\hline $\begin{array}{l}\text { Siltuximab } \pm \text { prednisone } \\
\text { Tocilizumab } \pm \text { prednisone } \\
\text { Rituximab* } \pm \text { prednisone }\end{array}$ & $\begin{array}{c}\text { Siltuximab } \\
\pm \text { HD methylprednisolone } \\
\text { Tocilizumab } \\
\pm \text { HD methyprednisolone } \\
\pm \text { cyclosporine** }\end{array}$ \\
\hline \multicolumn{2}{|c|}{ Second-line therapy } \\
\hline $\begin{array}{l}\text { Rituximab } \pm \text { prednisone } \\
\pm \text { immunomodulator } \\
\text { (thalidomide) }\end{array}$ & $\begin{array}{l}\mathrm{CVP} \pm \text { rituximab } \\
\mathrm{CHOP} \pm \text { rituximab }\end{array}$ \\
\hline $\begin{array}{l}\text { Thalidomide } \pm \text { prednisone } \\
\pm \text { cyclophosphamide } * * *\end{array}$ & \\
\hline Lenalidomide & \\
\hline Second-line drugs & \\
\hline Bortezomib & \\
\hline Cyclosporine & \\
\hline Sirolimus & \\
\hline Anti-IL-1 & \\
\hline
\end{tabular}

*According to CDCN recommendations, first-line rituximab is recommended when anti-IL-6/IL-6R antibodies are unavailable, or when short-term anti-CD20 therapy is considered as an alternative to long-term anti-IL 6 therapy; ${ }^{* *}$ combination of tocilizumab, HD-steroids, cyclosporine recommended by Japanese research group in iMCD-TAFRO therapy; $* * *$ TCP regimen [31] with an immunomodulatory drug in second-line of treatment or in first-line when anti IL-6/IL-6R are unavailable; NOS - not otherwise specified; TAFRO - thrombocytopenia, ascites, fibrosis, renal, organomegaly; IL-1 - interleukin 1; HD - high dose; CVP - cyclophosphamide, vincristine, prednisone; CHOP - cyclophosphamide, adriamycin, vincristine, prednisone

\section{Treatment of mild iMCD}

Patients who do not meet the criteria of severe iMCD (Table II), without obvious organ dysfunction, in relatively good general condition, with moderate severity of IL-6-dependent inflammatory symptoms (fatigue, anaemia, impaired exercise tolerance, peripheral oedema) may require treatment due to their deteriorating quality of life, expressed not only by the disturbance in performing daily activities, but also by limitations in their professional life. Clinically significant lymphadenopathy is a rare indication for treatment initiation. Based on the results of a phase II study (the only randomised, double-blind, placebo-controlled study), the CDCN recommends (category 1 ) first-line treatment with an anti-interleukin 6 (anti-IL-6) monoclonal antibody, siltuximab (11 mg/kg i.v. every three weeks) [23]. The safety of the drug was confirmed by the results of long-term observation in a previous phase I/II study, in which the median duration of treatment was 5.1 (range 3.4-7.2) years, with a median of 81 cycles administered (range $49-129$ ). In this study, as many as $74 \%$ of patients 
Table IV. Response criteria according to Castleman Disease Collaborative Network (CDCN) (2018) [23]

\begin{tabular}{|l|l|l|l|}
$\begin{array}{l}\text { Complete response } \\
(1+2+3)\end{array}$ & $\begin{array}{l}\text { 1. Laboratory workup: Hb, CRP } \\
\text { albumins, eGFR }\end{array}$ & $\begin{array}{l}\text { 2. Assessment of nodal lesions } \\
\text { according to Cheson }\end{array}$ & $\begin{array}{l}\text { 3. Evaluation of systemic symptoms: } \\
\text { fatigue, anorexia, fever, body weight* }\end{array}$ \\
\hline $\begin{array}{l}\text { CR } \\
\text { PR }\end{array}$ & $\begin{array}{l}\text { Cormal } \\
>50 \% \text { improvement from base- } \\
\text { line applies to all parameters }\end{array}$ & PR & $\begin{array}{l}\text { Complete resolution of symptoms } \\
\text { Improvement in all symptoms } \\
\text { (but not complete resolution) }\end{array}$ \\
\hline SD & $\begin{array}{l}\text { Improvement }<50 \% \text { or deterio- } \\
\text { ration }<25 \% \text { of all parameters }\end{array}$ & $\begin{array}{l}\text { Does not meet CR, PR or PD } \\
\text { criteria }\end{array}$ & $\begin{array}{l}\text { Improvement in at least one symptom } \\
\text { (but not all) }\end{array}$ \\
\hline $\begin{array}{l}\text { Deterioration of }>25 \% \text { of one } \\
\text { parameter }\end{array}$ & PD & $\begin{array}{l}\text { Worsening of at least one symptom as- } \\
\text { sessed at least twice four weeks apart }\end{array}$
\end{tabular}

*Symptom Severity Score assessed by Common Terminology Criteria of Adverse Events (CTCAE) version 4, improvement in fatigue, appetite and fever symptoms is $\geq 1$ grade CTC reduction from baseline, weight gain $\geq 5 \%$ increase to baseline values; $\mathrm{Hb}$ - haemoglobin; CRP - C-reactive protein; eGFR - estimated glomerular filtration rate; $\mathrm{CR}$ - complete remission; PR - partial remission; SD - stable disease; $\mathrm{PD}$ - progressive disease

stayed on treatment for at least four years [24, 25]. The phase II study included 79 patients (iMCD) randomised to siltuximab or a placebo arm. In the siltuximab group, 34\% nodal regression responses (complete or partial remission maintained for at least 18 weeks) were achieved, but no remission was observed in the placebo group. Benefit from the administration of siltuximab, defined as a significant improvement or relief of symptoms related to the disease, was observed in $60 \%$ of patients [26].

Data analysis and clinical experience indicate that the greatest benefit from the administration of anti-IL-6 treatment is achieved in patients with severe cytokine-dependent symptoms, defined as the presence of laboratory abnormalities (high CRP, ESR, fibrinogen, hypergammaglobulinemia, significant anaemia, hypoalbuminemia). Final analysis for the predictive model of response to siltuximab identified four important parameters: elevated fibrinogen levels, CRP, hypergammaglobulinemia, and decreased haemoglobin levels. The level of IL-6 alone did not influence the response [27]. The expected effect of siltuximab on nodal regression requires time, as this drug has no direct cytotoxic effect. In the phase II study, mean time to obtain overall response rate (ORR), defined as CR or PR, was five months [26]. It is recommended that in the early stages of treatment, biochemical parameter monitoring (CRP, ESR, albumin) and the improvement or resolution of disease-related symptoms should be used to evaluate treatment response. Nodal remission evaluation (using diagnostic imaging) should not be performed earlier than three months after treatment initiation [14, 23]. Patients who benefit from siltuximab treatment should continue the treatment, because there is a risk of relapse after treatment cessation. In a small group of patients receiving the drug for a long time, the intervals between doses were safely extended from three to six weeks, but the final effect of such a procedure requires longer observation. Many years of follow-up of patients receiving siltuximab did not reveal any significant treatment-related complications. The most frequently observed side effects include lipid disorders (hypertriglyceridemia, hypercholesterolaemia), a slight decrease in platelet count, and itching [25]. Based on the results of the phase II study, siltuximab has been approved for the treatment of IMCD in the United States and the European Union.

When siltuximab is unavailable, anti-interleukin 6 receptor monoclonal antibody (anti-IL-6R), tocilizumab ( $8 \mathrm{mg} /$ /kg b.w. i.v. every two weeks), is recommended by the $\mathrm{CDCN}$ as an alternative first-line treatment (category $2 \mathrm{~A}$ ). This drug has similar safety and efficacy profiles to siltuximab, but the evidence for its effectiveness is not supported by a randomised controlled trial. In a small, prospective, single-arm study (35 patients with iMCD), $86 \%$ of patients who continued treatment for at least five years benefited from tocilizumab. However, the effectiveness of the drug is determined here only by the percentage of patients who continued treatment (86\%), and objective response criteria were not shown [28]. The drug has been approved for the treatment of $\mathrm{iMCD}$ in Japan. It is not recommended to initiate anti-IL-6 (siltuximab) or anti-IL-6R (tocilizumab) therapy solely based on IL-6 levels. In the phase II study with siltuximab, several cases of patients with low or normal IL-6 levels who responded to treatment, as well as cases of patients with high levels of IL-6 without response to the antibody, were observed [26]. IL-6 concentration was not considered a criterion of response to the treatment because both siltuximab and tocilizumab can cause false elevations in IL- 6 levels even up to 24 months after the final dose of the drug [14, 23, 29].

Depending on the clinical situation, anti-IL-6 therapy can be administered in combination with corticosteroids. Prednisone (1 mg/k b.w. for 4-8 weeks) should be considered with a gradual dose reduction (category $2 \mathrm{~B}$ ). It is possible to use higher doses of steroids depending on the clinical need. It is not recommended to administer corticosteroids in monotherapy because, despite their initially high effectiveness ( $46 \%$ ORR), extended treatment is associated with a high rate of progression or requires a change of therapy due to side effects (54\% failures) [23]. 
If anti-IL6/anti-IL6R antibodies are unavailable, the alternative first-line treatment should consist of antiCD20 monoclonal antibody, rituximab $\left(375 \mathrm{mg} / \mathrm{m}^{2}\right.$ i.v., 4-8 doses), most often in combination with steroids (category 2B). Rituximab can be administered initially in weekly infusions (four doses) or at 2-3 weekly intervals (6-8 doses) until the major systemic symptoms (e.g. general symptoms, fever, malaise) resolve. According to the $\mathrm{CDCN}$ recommendations, rituximab is proposed as the first-line drug for patients who are considering the possibility of short-term therapy (4-8 cycles) as an alternative to long-term anti-IL-6 therapy [23]. Long-term maintenance treatment with rituximab is not recommended.

There is no conclusive evidence for the efficacy of rituximab in patients with idiopathic MCD, as the evaluation of its efficacy is mainly based on the experience with $\mathrm{HIV}+\mathrm{HHV}-8+\mathrm{MCD}$. The results of a retrospective analysis comparing the efficacy of siltuximab to that of rituximab for iMCD patients indicate that the anti-IL6 (siltuximab)-terated group had a significantly higher $\mathrm{CR}$ rate $(p=0.034)$ and progression-free survival (PFS). It is worth noting that in the siltuximab group, approximately $75 \%$ of objective responses (ORR), defined as regression of nodal lesions, were achieved. This is a much higher percentage compared to the registration phase II study (34\% ORR) [22]. The discrepancy in the obtained results is explained by the fact that the patients enrolled to the phase II study had less severe systemic symptoms, which made the placebo arm possible, while patients from the retrospective analysis constituted the 'real life' population presenting a typical broad spectrum of symptoms. This observation seems to confirm that anti-IL-6 efficacy is directly correlated with the severity of inflammatory symptoms.

First-line anti-IL-6/ anti-IL-6R treatment failure is observed in approximately $40-50 \%$ of patients. In these cases, it is recommended to use immunomodulatory or immunosuppressive drugs, possibly in combination with steroids and rituximab (category $2 \mathrm{~B}$ ). The most important immunomodulatory drug, thalidomide, is effective in monotherapy as well as in combination with rituximab. It lowers IL-6 expression and shows anti-angiogenic activity by lowering VEGF $[23,30]$. In 2019, the results of a prospective phase II study were published. The study aimed to evaluate the effectiveness of the TCP regimen based on the use of oral immunochemotherapy (TCP: thalidomide $100 \mathrm{mg}$ daily for two years, oral cyclophosphamide $300 \mathrm{mg} / \mathrm{m}^{2}$ on days $1,8,15$, and 22 in a 28-day cycle for one year, prednisone $1 \mathrm{mg} / \mathrm{kg}$ b.w. on days 1, 2, 8, 9, 15, 16, 22, 23 in a 28-day cycle for one year). A high percentage of objective responses (48\% ORR) was achieved in the group of previously untreated patients, with good treatment tolerance. Based on these results, TCD regimen can be considered as a first-line treatment, especially in countries where siltuximab or tocilizumab are unavailable [31].
The recommendations for the third and subsequent lines of treatment are not strictly defined. According to the CDCN, if iMCD does not meet the severe form criteria, classic chemotherapy regimens should be avoided [23]. It is recommended to use other immunomodulatory or immunosuppressive drugs, because their effectiveness is comparable to classic chemotherapy (ORR 69\%), but with significantly lower toxicity. The use of lenalidomide, bortezomib, cyclosporin $\mathrm{A}$, or an IL-1 $\beta$ receptor antagonist anakinra (superior control of IL-1 over IL-6) should be considered [14, 22, 23, 32-34]. The efficacy of the mTOR pathway antagonist sirolimus has also been reported. A study is currently underway to assess the efficacy of sirolimus in patients after treatment failure with anti-IL6 (NCT03933904) [19].

\section{Treatment of severe iMCD}

It is estimated that the severe form of $\mathrm{MMCD}$ accounts for about $10-20 \%$ of all CD cases. Clinically significant organ dysfunction (renal failure, massive oedema and exudates, respiratory failure, poor general condition) can be lifethreatening. This group of patients requires urgent use of high doses of steroids. It is recommended (category 1 ) to administer methylprednisolone at a dose of $500 \mathrm{mg} /$ day simultaneously with siltuximab, which in this situation may be administered once a week for the first month of intensive care $(11 \mathrm{mg} / \mathrm{kg}$ b.w. at days $1,8,15,22)$. The patients who benefit from the treatment should continue siltuximab therapy in the standard regimen (every three weeks). At the same time, it is recommended to gradually reduce the doses of steroids until their complete discontinuation as soon as possible. An alternative option is to use tocilizumab (category $2 A$ ). Due to the high risk of mortality in severe iMCD, it should be remembered that not all patients will respond to the treatment quickly. Some will not respond to the combination of anti-IL-6 with steroids. Careful daily monitoring of the patient's condition and laboratory parameters to evaluate the response is recommended. If the general condition deteriorates, or no improvement is observed after a week of siltuximab administration, conventional cytostatics in multidrug regimens typically used to treat non-Hodgkin's lymphoma, multiple myeloma or haemophagocytic lymphohistiocytosis (HLH) should be administered [23].

The most commonly used regimens include: CVP $\pm \mathrm{R}$ (cyclophosphamide, vincristine, prednisone \pm rituximab), $\mathrm{CHOP} \pm \mathrm{R}$ (cyclophosphamide, adriamycin, vincristine, prednisone/dexamethasone \pm rituximab), VDT-ACE-R (bortezomib, dexamethasone, thalidomide, adriamycin, cyclophosphamide, etoposide, rituximab), and HLH-scheme (etoposide, prednisone/dexamethasone, cyclosporine). Experience with the administration of these regimens is based on small groups of patients, and the evaluation of their effectiveness comes from retrospective analyses and case reports $[23,35]$. Attempts to perform autologous or allogenic transplantation are casuistic and, according to 
the recommendations of $C D C N$ experts, should be considered only as a last resort therapy (with the exception of POEMS-MCD with bone lesions as an indication for autologous transplantation) $[14,23]$.

Severe iMCD often meets the criteria for TAFRO syndrome. In such cases, the CDCN recommends the same procedure as for iMCD-NOS. It seems that cyclosporine may play an important role in the therapy of TAFRO, especially in cases of recurrent thrombocytopenia and ascites. The Japanese research group recommends the use of tocilizumab in combination with cyclosporine and high doses of steroids in patients with TAFRO syndrome [36]. The effectiveness of other immunosuppressants, including calcineurin inhibitor (tacrolimus) and mTOR inhibitor (sirolimus), has also been described [37, 38].

\section{Treatment response evaluation}

The criteria for evaluation of treatment response were standardised by the CDCN in 2017 (Table IV). Comprehensive evaluation of treatment response includes: 1) laboratory evaluation of inflammatory markers including four parameters: haemoglobin ( $\mathrm{Hb}), \mathrm{CRP}$, albumin, and eGFR; 2) regression of nodal lesions according to modified Cheson criteria [39]; and 3) assessment of clinical symptoms (fatigue, eating disorders, fever, body weight) according to the National Cancer Institute Common Terminology Criteria of Adverse Events scale [version 4 of the National Cancer Institute - Common Toxicity Criteria ( $\mathrm{NCl}-\mathrm{CTC}$ ) of adverse events $(A E)]$. CR requires normalisation of all biochemical parameters, PR is defined as improvement ranging from $50 \%$ to $99 \%$ of baseline, while stable disease (SD) is defined as an improvement of less than $50 \%$ of baseline or deterioration of any of the parameters $<25 \%$. Progressive disease (PD) is defined as a deterioration of any of the parameters by $>25 \%$ of baseline. Relief of all disease-related symptoms or improvement in their severity (but not complete resolution) as assessed by the Common Terminology Criteria of Adverse Events (CTCAE) scale indicates CR or PR in the assessment of systemic symptoms. In this case, progression means the deterioration of one of the symptoms observed on at least two consecutive visits, four weeks apart [23].

\section{CDCN Project, NCT02817997}

As part of the CDCN-supported project (NCT02817997), each patient can register their medical history at http:// //www.cdcn.org/accelerate. The purpose of the registry is to obtain demographic, clinical and laboratory data for further research into understanding this rare disease [14].

\section{Authors' contributions}

BO - concept and design of study, manuscript preparation and verification; JRJ - manuscript preparation and verification; KDCZ - manuscript preparation and verification; JW - manuscript preparation and verification. All authors approved the final version of this article.

\section{Conflict of interest}

BO - advisory role: EUSA Pharma. The other authors have no conflict of interest to declare.

\section{Financial support}

None.

\section{Ethics}

The work described in this article has been carried out in accordance with The Code of Ethics of the World Medical Association (Declaration of Helsinki) for experiments involving humans; EU Directive 2010/63/EU for animal experiments; Uniform requirements for manuscripts submitted to biomedical journals.

\section{References}

1. Castleman B, Towne VW. Case records of the Massachusetts General Hospital; weekly clinicopathological exercises founded by Richard C. Cabot. N Engl J Med. 1954; 251: 396-400.

2. Gaba AR, Stein RS, Sweet DL, et al. Multicentric giant lymph node hyperplasia. Am J Clin Pathol. 1978; 69(1): 86-90, doi: 10.1093/ /ajcp/69.1.86, indexed in Pubmed: 619617.

3. Lachant NA, Sun NC, Leong LA, et al. Multicentric angiofollicular lymph node hyperplasia (Castleman's disease) followed by Kaposi's sarcoma in two homosexual males with the acquired immunodeficiency syndrome (AIDS). Am J Clin Pathol. 1985; 83(1): 27-33, doi: 10.1093/ /ajcp/83.1.27, indexed in Pubmed: 3871303.

4. Soulier J, Grollet L, Oksenhendler E, et al. Kaposi's sarcoma-associated herpesvirus-like DNA sequences in multicentric Castleman's disease. Blood. 1995; 86(4): 1276-1280, indexed in Pubmed: 7632932.

5. Fajgenbaum DC, Fajgenbaum DC. Novel insights and therapeutic approaches in idiopathic multicentric Castleman disease. Blood. 2018; 132(22): 2323-2330, doi: 10.1182/blood-2018-05-848671, indexed in Pubmed: 30487129.

6. Fajgenbaum DC, Uldrick TS, Bagg A, et al. International, evidence-based consensus diagnostic criteria for HHV-8-negative/idiopathic multicentric Castleman disease. Blood. 2017; 129(12): 1646-1657, doi: 10.1182/blood-2016-10-746933, indexed in Pubmed: 28087540.

7. Takai K, Nikkuni K, Shibuya H, et al. [Thrombocytopenia with mild bone marrow fibrosis accompanied by fever, pleural effusion, ascites and hepatosplenomegaly] [Article in Japanese]. Rinsho Ketsueki. 2010; 51(5): 320-325, indexed in Pubmed: 20534952.

8. Simpson D. Epidemiology of Castleman disease. Hematol Oncol Clin North Am. 2018; 32(1): 1-10, doi: 10.1016/j.hoc.2017.09.001, indexed in Pubmed: 29157611.

9. Boutboul D, Fadlallah J, Chawki S, et al. Treatment and outcome of unicentric Castleman disease: a retrospective analysis of 71 cases. Br J Haematol. 2019; 186(2): 269-273, doi: 10.1111/bjh.15921, indexed in Pubmed: 31016730.

10. Dispenzieri A, Armitage JO, Loe MJ, et al. The clinical spectrum of Castleman's disease. Am J Hematol. 2012; 87(11): 997-1002, doi: 10.1002/ajh.23291, indexed in Pubmed: 22791417. 
11. Oksenhendler E, Boutboul D, Fajgenbaum D, et al. The full spectrum of Castleman disease: 273 patients studied over 20 years. Br J Haematol. 2018; 180(2): 206-216, doi: 10.1111/bjh.15019, indexed in Pubmed: 29143319.

12. Iwaki N, Fajgenbaum DC, Nabel CS, et al. Clinicopathologic analysis of TAFRO syndrome demonstrates a distinct subtype of HHV-8-negative multicentric Castleman disease. Am J Hematol. 2016; 91(2): 220-226, doi: 10.1002/ajh.24242, indexed in Pubmed: 26805758.

13. Bower M, Newsom-Davis T, Naresh K, et al. Clinical features and outcome in HIV-associated multicentric Castleman's disease. J Clin Oncol. 2011; 29(18): 2481-2486, doi: 10.1200/JC0.2010.34.1909, indexed in Pubmed: 21555697.

14. Dispenzieri A, Fajgenbaum DC. Overview of Castleman disease. Blood. 2020; 135(16): 1353-1364, doi: 10.1182/blood.2019000931, indexed in Pubmed: 32106302.

15. Nabel CS, Sameroff S, Shilling D, et al. Virome capture sequencing does not identify active viral infection in unicentric and idiopathic multicentric Castleman disease. PLoS One. 2019; 14(6): e0218660, doi: 10.1371/journal. pone.0218660, indexed in Pubmed: 31242229.

16. Liu AY, Nabel CS, Finkelman BS, et al. Idiopathic multicentric Castleman's disease: a systematic literature review. Lancet Haematol. 2016; 3(4): e163-e175, doi: 10.1016/S2352-3026(16)00006-5, indexed in Pubmed: 27063975.

17. Li Z, Lan X, Li C, et al. Recurrent PDGFRB mutations in unicentric Castleman disease. Leukemia. 2019; 33(4): 1035-1038, doi: 10.1038/ /s41375-018-0323-6, indexed in Pubmed: 30607019.

18. You L, Lin Q, Zhao J, et al. Whole-exome sequencing identifies novel somatic alterations associated with outcomes in idiopathic multicentric Castleman disease. Br J Haematol. 2020; 188(5): e64-e67, doi: 10.1111/bjh.16330, indexed in Pubmed: 31863597.

19. Fajgenbaum $D C$, Langan $R A$, Japp $A S$, et al. Identifying and targeting pathogenic PI3K/AKT/mTOR signaling in IL-6-blockade-refractory idiopathic multicentric Castleman disease. J Clin Invest. 2019; 129(10): 4451-4463, doi: 10.1172/JCl126091, indexed in Pubmed: 31408438.

20. El-Osta HE, Kurzrock R. Castleman's disease: from basic mechanisms to molecular therapeutics. Oncologist. 2011; 16(4): 497-511, doi: 10.1634/theoncologist.2010-0212, indexed in Pubmed: 21441298.

21. Talat N, Belgaumkar AP, Schulte KM. Surgery in Castleman's disease: a systematic review of 404 published cases. Ann Surg. 2012; 255(4): 677-684, doi: 10.1097/SLA.0b013e318249dcdc, indexed in Pubmed: 22367441.

22. Yu Li, Tu M, Cortes J, et al. Clinical and pathological characteristics of HIV- and HHV-8-negative Castleman disease. Blood. 2017; 129(12): 1658-1668, doi: 10.1182/blood-2016-11-748855, indexed in Pubmed: 28100459.

23. Rhee Fv, Voorhees P, Dispenzieri A, et al. International, evidence-based consensus treatment guidelines for idiopathic multicentric Castleman disease. Blood. 2018; 132(20): 2115-2124, doi: 10.1182/blood-2018-07-862334.

24. van Rhee F, Casper C, Voorhees PM, et al. A phase I, open-label study of siltuximab, an anti-IL-6 monoclonal antibody, in patients with B-cell non-Hodgkin lymphoma, multiple myeloma, or Castleman disease. Clin Cancer Res. 2013; 19(13): 3659-3670, doi: 10.1158/10780432.CCR-12-3349, indexed in Pubmed: 23659971.

25. van Rhee F, Casper C, Voorhees PM, et al. A phase 2, open-label, multicenter study of the long-term safety of siltuximab (an anti-interleukin-6 monoclonal antibody) in patients with multicentric Castleman disease. Oncotarget. 2015; 6(30): 30408-30419, doi: 10.18632/oncotarget.4655, indexed in Pubmed: 26327301.
26. van Rhee F, Wong RS, Munshi N, et al. Siltuximab for multicentric Castleman's disease: a randomised, double-blind, placebo-controlled trial. Lancet Oncol. 2014; 15(9): 966-974, doi: 10.1016/S14702045(14)70319-5, indexed in Pubmed: 25042199.

27. Morra DE, Pierson S, Shilling D, et al. Predictors of response to anti-IL6 monoclonal antibody therapy (siltuximab) in idiopathic multicentric Castleman disease: secondary analyses of phasellclinical trial data. Br J Haematol. 2018; 184(2): 232-241, doi: 10.1111/bjh.15588, indexed in Pubmed: 30203839.

28. Nishimoto $\mathrm{N}$, Honda $\mathrm{O}$, Sumikawa $\mathrm{H}$, et al. A long-term (5-year) sustained efficacy of tocilizumab for multicentric Castleman's disease and the effect on pulmonary complications. Blood. 2007; 110(11): 646-646, doi: 10.1182/blood.v110.11.646.646.

29. Nishimoto N, Terao K, Mima T, et al. Mechanisms and pathologic significances in increase in serum interleukin-6 (IL-6) and soluble IL-6 receptor after administration of an anti-IL-6 receptor antibody, tocilizumab, in patients with rheumatoid arthritis and Castleman disease. Blood. 2008; 112(10): 3959-3964, doi: 10.1182/ /blood-2008-05-155846, indexed in Pubmed: 18784373.

30. Ramasamy K, Gandhi S, Tenant-Flowers M, et al. Rituximab and thalidomide combination therapy for Castleman disease. Br J Haematol. 2012; 158(3): 421-423, doi: 10.1111/j.1365-2141.2012. 09157.x, indexed in Pubmed: 22583139.

31. Zhang Lu, Zhao AL, Duan MH, et al. Phase 2 study using oral thalidomide-cyclophosphamide-prednisone for idiopathic multicentric Castleman disease. Blood. 2019; 133(16): 1720-1728, doi: 10.1182/ /blood-2018-11-884577, indexed in Pubmed: 30760451.

32. El-Osta H, Janku F, Kurzrock R. Successful treatment of Castleman's disease with interleukin-1 receptor antagonist (Anakinra). Mol Cancer Ther. 2010; 9(6): 1485-1488, doi: 10.1158/1535-7163.MCT-100156, indexed in Pubmed: 20501803.

33. Zhou $X$, Wei J, Lou $Y$, et al. Salvage therapy with lenalidomide containing regimen for relapsed/refractory Castleman disease: a report of three cases. Front Med. 2017; 11(2): 287-292, doi: 10.1007/ /s11684-017-0510-2, indexed in Pubmed: 28367597.

34. Khan AA, Siraj F, Bhargava M, et al. Successful treatment of multicentric Castleman's disease accompanying myeloma with bortezomib. BMJ Case Rep. 2012; 2012, doi: 10.1136/bcr-2012-007646, indexed in Pubmed: 23264156.

35. Zhu SH, Yu YH, Zhang Y, et al. Clinical features and outcome of patients with HIV-negative multicentric Castleman's disease treated with combination chemotherapy: a report on 10 patients. Med Oncol. 2013; 30(1): 492, doi: 10.1007/s12032-013-0492-0, indexed in Pubmed: 23400962.

36. Masaki Y, Kawabata H, Takai K, et al. Proposed diagnostic criteria, disease severity classification and treatment strategy for TAFRO syndrome, 2015 version. Int J Hematol. 2016; 103(6): 686-692, doi: 10.1007/s12185-016-1979-1, indexed in Pubmed: 27084250.

37. Shirai T, Onishi A, Waki D, et al. Successful treatment with tacrolimus in TAFRO syndrome: two case reports and literature review. Medicine (Baltimore). 2018; 97(23): e11045, doi: 10.1097/ /MD.0000000000011045, indexed in Pubmed: 29879072.

38. Fajgenbaum D, Shilling D, Partridge HL, et al. Prolonged remission achieved in a relapsing idiopathic multicentric castleman disease patient with a novel, targeted treatment approach [abstract]. Blood. 2017; 130(Suppl 1): 3593, doi: 10.1182/blood.V130.Suppl_1.3593.3593.

39. Cheson BD, Horning SJ, Coiffier B, et al. Report of an international workshop to standardize response criteria for non-Hodgkin's lymphomas. NCl Sponsored International Working Group. J Clin Oncol. 1999; 17(4): 1244, doi: 10.1200/JC0.1999.17.4.1244, indexed in Pubmed: 10561185. 